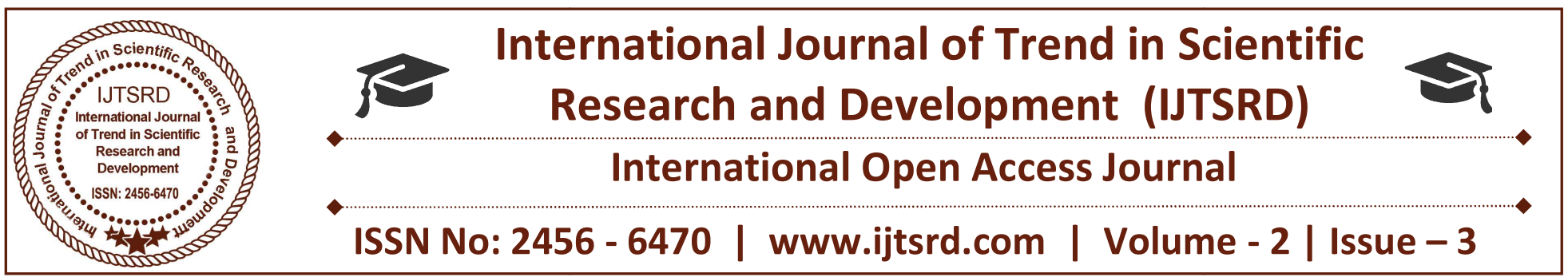

\title{
MIMO-OFDM's BER and Design Performance for Wireless Broadband Communications
}

\author{
Shuja Behna, Neetu Sikarwar \\ Institute of Engineering, Jiwaji University, \\ Gwalior, Madhya Pradesh., India
}

\begin{abstract}
To get high data rates, speed and immediate amplify in range and reliability without consuming more radio frequency require MIMO-OFDM for wireless broadband communication. In this paper, we investigate the performance of MIMO-OFDM using diverse modulation schemes are used to encode and decode the data stream in wireless communication over AWGN channel for the indefinite transmitter and known receiver. In exacting we relate MIMO detection methods based on V-BLAST (Vertical Bells Lab Layered Space Time) planning to develop spectral efficiency.
\end{abstract}

Keywords: Orthogonal Frequency Division Multiplexing (OFDM), Vertical Bells Lab Layered Space Time(V-BLAST), Multiple Input Multiple Output (MIMO), Bit Error Rate (BER).

\section{INTRODUCTION}

Nowadays, wireless communication systems square measure enjoying the crucial role. Initially, wireless systems were in the main designed to support voice. Later these square measure accustomed transfer the information, they gain quality thanks to their simple use and quality. All wireless technology faces the challenges of signal attenuation, multipath, increasing interference and restricted spectrum. Orthogonal Frequency Division Multiplexing (OFDM) plays an important role and cut back receiver complexness in wireless broadband systems however during this case synchronization and channel estimation square measure vital, and it's replaced by Multiple Input Multiple Output-Orthogonal Frequency
Division Multiplexing (MIMO-OFDM) that could be a multi-user OFDM that enables multiple accesses that theme that mixes TDM and FDM on identical channel, wide for succeeding generation wireless communication systems like WLAN,WMAN, WiMAX and 3G-LTE normal so as to accommodate several users within the same channel at identical time. The utilization of MIMO technology together with OFDM, i.e., MIMO-OFDM, therefore, looks to be a horny answer for future broadband wireless systems. however, this MIMO system having quick framing rate of the order of $1-2 \mu$ s are contaminated by international intelligence agency once operational in an associate atmosphere having a typical time delay unfold of two hundred $\mu$ s. So associated international intelligence agency worth of 200/2 = 100 is the associate undesirable multi-path result for the important MIMO system. Thus MIMO cannot reach zero international intelligence agencies and hence can't be utilized alone. The OFDM based mostly multi-carrier approach could also be an associate enabler for the MIMO broadband operation that the quick frames square measure bogged down 1st and regenerate too many slow sub frames and modulated to multiple carriers of OFDM. OFDM-MIMO is, therefore, a helpful technology which might be explored each for communication and remote sensing (radar).

Recently, IEEE 802.11n task cluster was shaped with a goal of accelerating the appliance outturn by creating changes within the PHY and Mackintosh layer. The main challenge within the physical layer is that the uses of multiple transmit and receive antennas and OFDM modulation that contains OFDM 
modulation Furthermore as subcarrier allocation. Therefore, it's important to focus additional attention on wireless communication technology. OFDM usually uses the next FFT size and divides the out their subcarriers into logical teams referred to as subchannels. in contrast to OFDM that transmits a similar quantity of energy in every subcarrier, OFDM could transmit completely different amounts of energy in every sub-channel i.e., users may additionally occupy quite one sub channel relying upon their Quality of Service (QoS).

\section{V-Blast Architecture}

The structure of the V-BLAST systems is described in fig.1Notation: Vector symbol a: (a1, a2, a3, a4... $\left.A_{M}\right)^{T}$, No. Of $T x=M_{T}$, No. of $R x=M_{R}$.

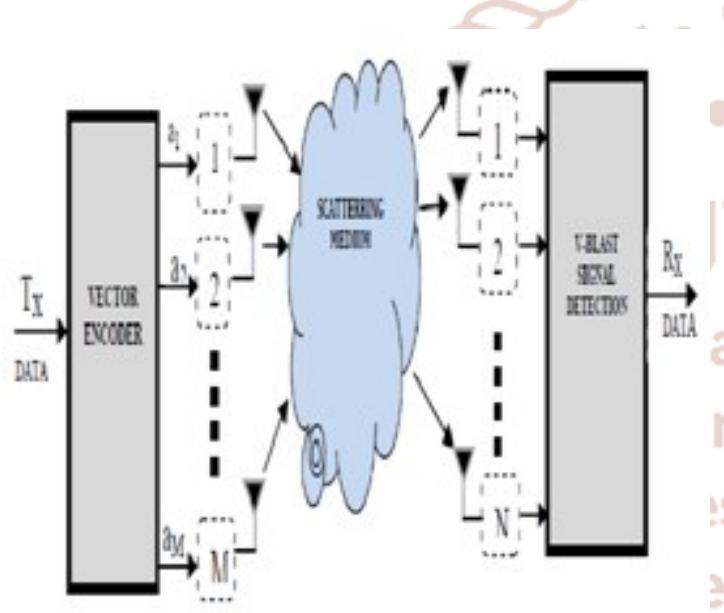

Figure 1: V-BLAST Architecture

A single information stream is de-multiplexed into $M$ sub streams, and every sub stream is then encoded into symbols and fed to its various transmitters. Transmitters $1-\mathrm{M}_{\mathrm{T}}$ operate co-channel at the image rate $1 / \mathrm{T}$ symbols/sec, with synchronic image temporal arrangement. Every transmitter is itself a normal QAM transmitter. The assembly of transmitters includes, in effect, a vector -appreciated transmitter, wherever fundamentals of each transmitted $\mathrm{M}_{\mathrm{T}}$ vector area unit symbols drawn from a QAM constellation. We have a tendency to assume that very same constellation is employed for every sub stream, which transmissions area unit organized into bursts of $\mathrm{L}$ symbols. The facility launched by every transmitter is proportional to $1 / \mathrm{M}_{\mathrm{T}}$ in order that the overall radiated power is constant and freelance of $\mathrm{M}_{\mathrm{T}}$.

\section{ExpertImental Setup for OFDM- MIMO (V-Blast) Systems}

Assuming an AWGN channel with the highest delay spread of $75 \mathrm{~ns}$, great channel awareness at the receiver and perfect synchronization, no awareness of the channel at the transmitter and employ interleaving. The modulation schemes employed are BPSK, QPSK and 16 QAM. The bandwidth of an IEEE 802.11a system is $20 \mathrm{MHz}$. There is 256 sub-carriers in each OFDM symbol. These mark for an inter-carrier spacing $\Delta$ fof $20 \mathrm{X} 106 / 256=781.25 \mathrm{KHz}$.

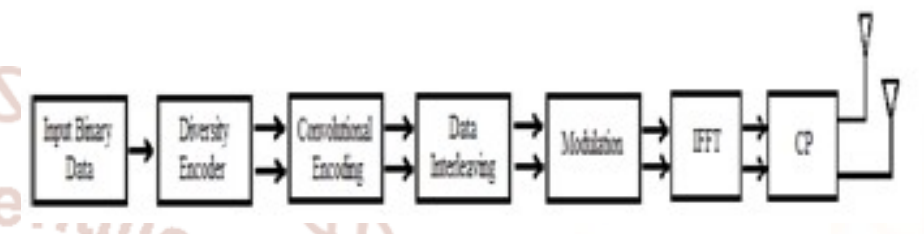

\section{(A) Transmitter}

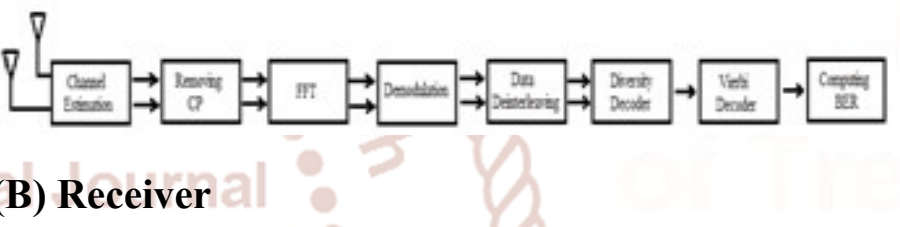

Figure 2: Block Diagram of OFDM-MIMO (VBlast) System

The binary input file is first sent to the variety encoder. In diversity encoder spatial multiplexing is applied it prevents from a protracted sequence of 0's and 1 's. This splits the info into orthogonal streams. Within the figure, we have a tendency to square measure considering $2 \times 2,2 \times 3,2 \times 4,4 \times 4$ systems. The primary stream is to the highest, second stream to the second and it's continued to different streams likewise severally. Here they're reborn from serial to parallel. Therefore the subcarriers square measure obtained. The subcarriers square measure then given to the convolution encoder. It's used for real time error correction. It's done by combining the mounted range of inputs. The input bits square measure keeps during a mounted length shift registers and that they square measure combined with the assistance of mod-2 adders. Associate degree input sequence associate degree contents of shift registers perform modulo-two addition when info sequence is shipped to shift registers so an output sequence is obtained. It's wont to improve BER and to cut back the high peak to average power magnitude relation that is a gift in OFDM. The actual customary for this encoder is $(2$, $1,7)$. The opposite rate $1 / 2$ is achieved by puncturing 
the output of this encoder. Puncturing involves deleting coded bits from output information sequence, such magnitude relation of un-coded bits to coded bits is bigger the mother code. The signal is then sent to the info interleaving. The concept of interleaving is to disperse a block of information in frequency so the whole block will expertise the deep fade within the channel. This prevents the burst errors at the receiver. Otherwise, the convolution decoder won't perform alright in victimisation BPSK, QPSK, and 16QAM schemes. They given to IFFT and append to the CP. therefore the data is transmitted in packets. The receiver is that the precise inverse method when the incoming packets square measure received. Diversity decoder converts parallel sub streams to serial type. The serial type is given to Viterbi decoder and is generally applied to convolution encoder and it uses the most probability decipherment technique. Clamorous channels cause bit errors at the receiver. The Viterbi rule estimates actual bit sequence victimisation trellis diagram. Then the $\mathrm{BER}$ is computed.

\section{IV.SIMULATION}

The on top of the system was simulated within the MATLAB.As we all know that the system is thought receiver and unknown transmitter the knowledge from the transmitter to receiver was received within the variety of packet or frame. The received packets could also be lost or embrace errors attributable to a loud channel. A performance analysis is finished for various Modulation schemes and for various transmits and receives components. We tend to transmit our knowledge by exploitation OFDM technique during which an oversized variety of closely spaced orthogonal subcarriers is employed to hold knowledge. Every Carrier is modulated and demodulated with modulation schemes. The encoded knowledge is responded to Gaussian wherever Additive White Gaussian noise (AWGN) is superimposed. There are unit some restrictions and downsides in digital wireless communication systems between transmitter and receiver wherever received signals hit the receiver with totally different power and time delay because of reflection, optical phenomenon and scattering effects. For this reasons, Bit Error Rate (BER) worth is comparatively high. During this condition, the digital wireless communication systems won't perform well.BER is that the basic parameter to access the standard.BER is just outlined as a variety of error bits/Number of total bits. Noise in transmission medium disturbs the signal and causes knowledge corruptions. The relation between signal and noise is delineated with SNR (signal-to-noise ratio).SNR is outlined as signal power/Noise power. SNR is reciprocally proportional with BER. The less the BER result's higher the SNR and also the higher communication quality. We tend to note that because the diversity order will increase the performance of V-Blast improves, that is to be expected. The variety order at the receiver is over adult male $-\mathrm{M}_{\mathrm{T}}+1$ and less than $\mathrm{M}_{\mathrm{R}}$. By exploitation FFT approach because the variety of subcarriers will increase the higher is accuracy because of a high variety of points. The information rate also will increase.

I) Compared with three modulation schemes, QPSK, BPSK and sixteen QAM with keeping the sending components fastened and ranging receiving components are shown in Fig 3, 4, 5. BER is diverse faintly since of its acceptance diversity method.

II) Performance of BPSK will improve BER and rate, at a little price of SNR. It operates between 4 and $6 \mathrm{~dB}$.

III) Performance of QPSK is most excellent than BPSK for steady information calculate however the information rate is going to be doubled. It operates between 8 and $10 \mathrm{~dB}$

IV) Performance of 16QAM has top SNR when put then with QPSK; BPSK. It operates between 10 and $14 \mathrm{~dB}$.

V) The $4 \times 4$ transmit and receiving elements as shown in fig 6 has better SNR in 16QAM when compared to other SNR.

Bit error probability curve for 16-QAM, QPSK and BPSK Modulation using OFDM Tx $=2 \mathrm{Rx}=2$

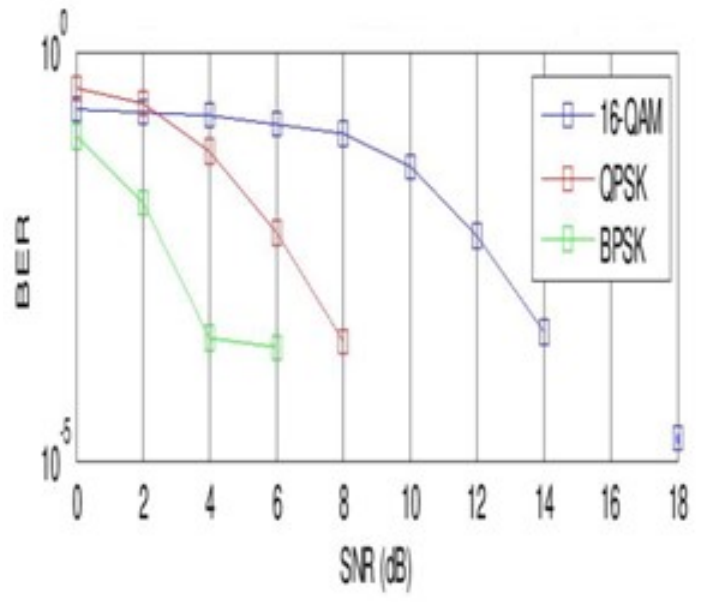

Figure 3: Performance of $2 \times 2$ 
Parameters: No. of FFT Points=256;

Channel=AWGN; No. Of Data Carriers $=256$

Bit error probability curve for 16-QAM,QPSK and BPSK Modulation using OFDM Tx=2 Rx $=3$

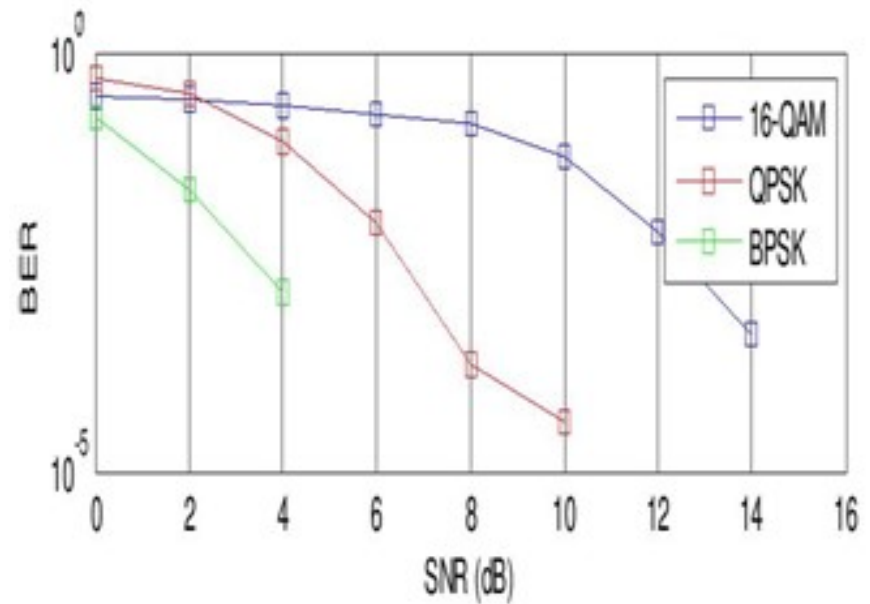

Figure 4: Performance of $2 \times 2$

Parameters: No. of FFT Points=256;

Channel=AWGN; No. Of Data Carriers $=256$

Bit error probability curve for 16-QAM,QPSK and BPSK Modulation using OFDM Tx $=2 \mathrm{Rx}=4$

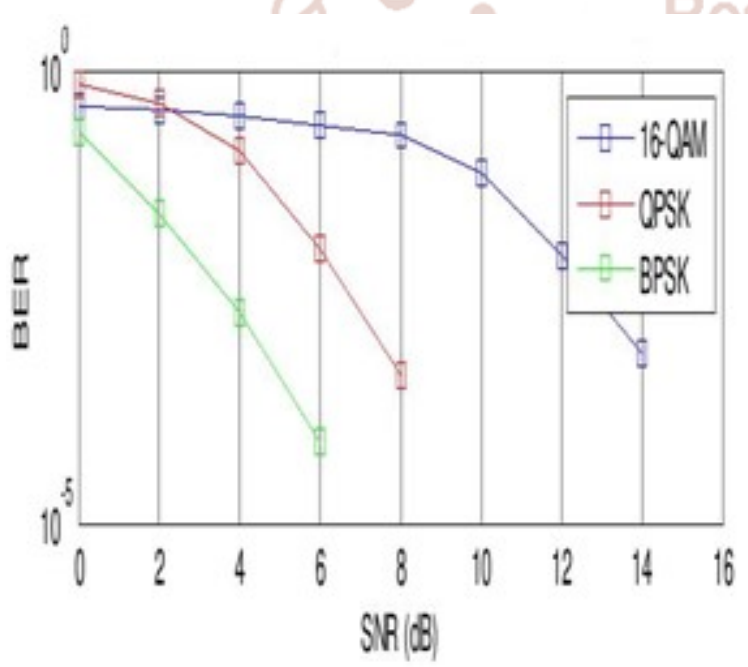

Figure 5: Performance of $2 \times 2$

Parameters: No. of FFT Points $=256$;

Channel=AWGN; No. Of Data Carriers $=256$

Bit error probability curve for 16-QAM,QPSK and BPSK Modulation using OFDM Tx $=4 \mathrm{Rx}=4 \mathrm{SNR}$

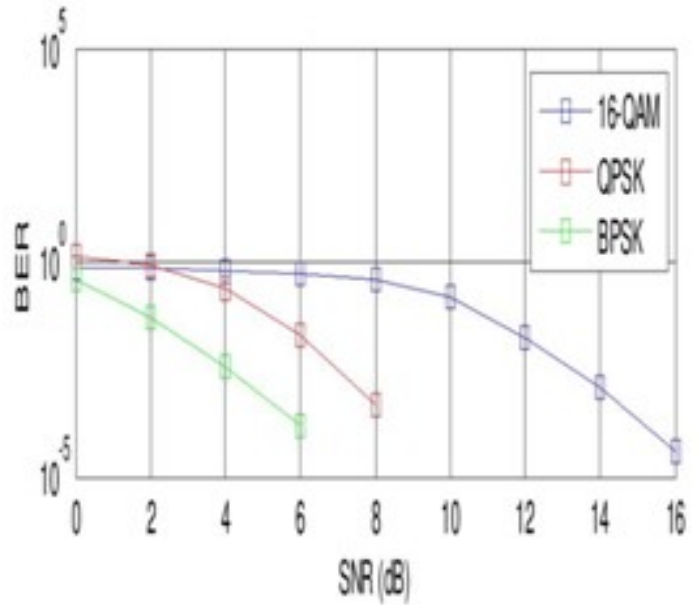

Figure 6: Performance of 2x2

Parameters: No. of FFT Points $=256$;

Channel=AWGN; No. Of Data Carriers $=256$

\section{CONCLUSION}

In this paper, we have a tendency to compare the performance of OFDM-MIMO (V-BLAST) in terms of BER victimisation totally different modulation schemes by variable each transmission and receiving components on AWGN channel. It's found that by employing a V-BLAST technique we will improve spectral potency.

\section{REFERENCES}

1. WiMAX Forum, Krishna Ramadas and Raj Jain, "WiMAX System Evaluation Methodology" version 2.1 July 2008 .

2. Foschini, G. J., "Layered space-time architecture for wireless communication in a fading environment when using multi-element antennas", Bell Labs Technical Journal, 1996.

3. IEEE 2009 NirmalenduBikas Sinha "Hybrid Technology using OFDM for Next Generation Broad band Mobile Radio Communications".

4. Zhangyong $\mathrm{Ma}$ and Young-il-Kim,"A Novel OFDM receiver in Flat Fading Channel", IEEE Confrence on advanced Communication technology, ICACT, Vol..2, pp.1052-54, 2005. [5] H.B olcskei, D.Gesbert, and A.J.Paulraj,"On the capacity of OFDM-based spatial multiplexing systems, IEEETrans.Commun" vol.50, no.2, pp.225-234, Feb.2002. 